EESTI NSV TEADUSTE AKADEEMIA TOIMETISED.

FOUSIKA. MATEMAATIKA

ИЗВЕСТИЯ АКАДЕМИИ НАУК ЭСТОНСКОИ ССР. ФИЗИКА. МАТЕМАТИКА

PROCEEDINGS OF THE ACADEMY OF SCIENCES OF THE ESTONIAN SSR. PHYSICS. MATHEMATICS

$1984,33,2$

Л. КИВИСТИК

удК 519.854 .62

\title{
ОБОБЩЕННОЕ ПРАВИЛО ВЫБОРА ПРОИЗВОДЯЩЕЙ СТРОКИ ДЛЯ ПОЛНОСТЬЮ ЦЕЛОЧИСЛЕННОГО АЛГОРИТМА ГОМОРИ
}

\author{
(Представил Н. Алумяэ)
}

Для полностью целочисленного алгоритма Гомори предлагается новое, более гибкое по сравнению с известными правило выбора производящей строки, которое называется обобщенным правилом. Доказывается конечность алгоритма при использовании обобщенного правила.

1. Постановка задачи и вводные замечания. Рассмотрим задачу полностью целочисленного линейного программирования в стандартной форме: максимизировать функцию

$$
x_{0}=a_{00}+\sum_{j \in J} a_{0 j}\left(-x_{j}\right)
$$

при условиях

$$
\begin{gathered}
x_{i}=a_{i 0}+\sum_{j \in J} a_{i j}\left(-x_{j}\right) \geqslant 0 \quad(i \in I), \\
x_{j}=-1\left(-x_{j}\right) \geqslant 0 \quad(j \in J), \\
x_{j} \text { - целое } \quad(j=0,1, \ldots, n),
\end{gathered}
$$

где $I$ - множество базисных, а $J$ - небазисных переменных, причем $I \cup J=\{1,2, \ldots, n\}$.

Для $j=0$ и $j \in J$ введем обозначение

$$
A_{j}=\left(a_{0 j}, a_{1 j}, \ldots, a_{n j}\right)^{\mathrm{T}},
$$

где $a_{k j}=0$, если $k \in J \backslash\{j\}$, и $a_{j j}=-1$, если $j \in J$.

Матрица $A=\left(a_{i j}\right)=\left(A_{j}\right) \quad(j \in\{0\} \cup J)$ обычно называется симплексной таблицей и векторы $A_{j}$ - ее столбцами.

Предположим, что $a_{i j}$ - целые числа $(i \in\{0\} \cup I ; j \in\{0\} \cup J)$ и симплексная таблица рассматриваемой задачи находится в $l$-нормальной форме, т. е. $A_{j}>0$ при всех $j \in J$. Тогда для решения задачи (1)-(4). применим' полностью целочисленный (или третий) алгоритм Гомори $\left[{ }^{1-3}\right]$. Напомним, что в этом алгоритме на каждой итерации выбирается ограничение

$$
x_{k}=a_{k 0}+\sum_{j \in J} a_{k j}\left(-x_{j}\right) \geqslant 0
$$

с $a_{k 0}<0$, называемое производящим, и по нему строится новое ограничение, т. н. отсечение

$$
x_{n+i}=\left[\frac{a_{k 0}}{\lambda}\right]+\sum_{j \in J}\left[\frac{a_{k j}}{\lambda}\right]\left(-x_{j}\right) \geqslant 0,
$$

которое прибавляется к системе (2)-(3). При этом для сохранения 
$l$-нормальности и целочисленности симплексной таблицы ведущий столбец $A_{l}$ следует определить из условия

$$
A_{l}=\underset{j: a_{k l}<0}{\operatorname{lex}} \min _{j}
$$

н затем число $\lambda>0$ - по соответствующим правилам $\left[{ }^{1-3}\right]$.

После каждой симплексной итерации вид задачи (1)-(4) сохраняется. Поэтому мы можем истолковать элементы $a_{i j}$ и столбцы $A_{j}$ симплексной таблицы (а также множества $I$ и $J$ ) как текущие. Однако для облегчения некоторых рассуждений введем индекс шага итерации и обозначим $a_{i j}$ и $A_{j}$ после $t$-й итерации также через $a_{i j}{ }^{t}$ и $A_{j}{ }^{t}$ соответственно.

Как известно $\left[{ }^{1,2}\right]$, имеют место лексикографические неравенства

$$
A_{0}^{0}>A_{0}^{1}>\ldots>A_{0}^{t}>A_{0}^{t+1}>\ldots \geq \bar{X}
$$

где $\bar{X}=\left(\bar{x}_{0}, \bar{x}_{1}, \ldots, \bar{x}_{n}\right)^{\mathrm{T}}-$ любое расширенное допустимое решение (в предположении, что такое существует).

В качестве производящего ограничения (5) обычно выбирают одно из ограничений (2), т. е. обычно $k \in I$. Но производящим может быть также т. н. замещающее ограничение

$$
\sum_{i \in I} a_{i 0} y_{i}+\sum_{j \in J}\left(\sum_{i \in I} a_{i j} y_{i}\right)\left(-x_{j}\right) \geqslant 0,
$$

где $y_{i} \geqslant 0(i \in I)$, т. е. ограничение (5) с $a_{k j}=\sum_{i \in I} a_{i j} y_{i}$ (при этом считаем, что $k>n)$. Р. Е. Гомори $\left[{ }^{1}\right]$ рассматривает также замещающие ограничения, которые строятся постепенно как линейные комбинации уже построенного отсечения и некоторого ограничения из системы (2). В настоящей заметке в качестве производящего может выступать любое ограничение типа (5), получаемое как следствие из условий (2)-(4).

Обычно для выбора производящего ограничения или, что то же самое, производящей строки в симплексной таблице $A$ пользуются правилом, заданным Р. Е. Гомори [ $\left.{ }^{1}\right]$ : всегда следует выбирать первую сверху строку $(k \neq 0)$, содержащую отрицательный свободный член $a_{k 0}$. Будем это правило называть о с н о в ы м.

Основное правило обеспечивает конечность полностью целочисленного алгоритма Гомори при условии, что задача (1)-(4) имеет по крайней мере одно допустимое решение [ $\left.{ }^{1}\right]$. Оно обеспечивает конечность также модификации этого алгоритма, предложенной Ю. Ю. Финкельштейном ([ $\left.{ }^{2}\right]$, с. $\left.184-186\right)$. Часто под третьим алгоритмом Гомори понимается именно такой его вариант, который использует основное правило (напр., $\left[{ }^{2}\right]$ ).

2. Обобщенное правило. Основное правило имеет тот недостаток, что оно не позволяет выбирать замещающего ограничения в качестве производящего. Однако именно подходяще построенные замещающие ограничения часто существенно ускоряют алгоритм. Кроме основного правила у Р. Е. Гомори [ $\left.{ }^{1}\right]$ имеется еще одно, гарантирующее конечность алгоритма. Оно позволяет циклически выбирать все строки с $a_{k 0}<0$ (в том числе и замещающие, хотя это явно не сказано). Однако желательно иметь такое правило, которое позволяло бы всегда выбирать производящую строку по ее качеству, а не по расположению в симплексной таблице. Тогда мы могли бы использовать на каждой итерации замещающее ограничение, если только оно достаточно хорошее. Такое правило содержится в нижеследующей теореме. При этом целесообразно ввести следующее понятие.

Будем говорить (ср. $\left[{ }^{1}\right]$, с. 235$)$, что вектор $A_{j}=\left(a_{0 j}, a_{1 j}, \ldots, a_{n j}\right)^{\mathrm{T}}$ 
имеет степен в вы рожденности $d$, если $a_{0 j}=\ldots \cdot=a_{d-1, j}=0$ и $a_{d j} \neq 0$.

Т е о р е м а. Если задача (1)-(4), симплексная таблица которой находится в l-нормальной форме, имеет хотя бы один план, то для обеспечения конечности полностью ьелочисленного алгоритма Гомори достаточно выбирать на каждой итерации в качестве производящего такое (заданное или замещающее) ограничение, при котором степень вырожденности д ведущего столбца (7) меньше чем

$$
k_{0}=\min \left\{i \in I \mid a_{i 0}<0\right\} .
$$

Д ок аз а тельство. Если симплексная таблица не находится в допустимой форме, то такое ограничение всегда существует. Действительно, так как в силу $A_{l}>0$ выполняется неравенство $d \leqslant k_{0}-1$, то таким является ограничение (5) с индексом $k=k_{0}$.

Пусть $\left(\bar{x}_{1}, \ldots, \bar{x}_{n}\right)^{\mathrm{T}}-$ некоторый план задачи $(1)-(4)$ и $\bar{x}_{0}-$ значение целевой функции (1) при этом плане. Тогда, в силу (8), $a_{00}{ }^{t} \geqslant \bar{x}_{0}$ при любом $t$. С увеличением $t$ значение $a_{00} t$ может только уменьшаться и если оно уменьшается, то на целое число. Поэтому, даже если алгоритм бесконечен, начиная с некоторого $t_{0}$ величина $a_{00}{ }^{t}$ должна оставаться неизменной, т. е. $a_{00}{ }^{t+1}=a_{00}{ }^{t}$ при $t \geqslant t_{0}$ (иначе $a_{00}{ }^{t}$ будет меньше $\left.\bar{x}_{0}\right)$. Дальше рассмотрим $a_{10}{ }^{t}$ при $t>t_{0}$. Если было бы $a_{10}{ }^{t}<0$, то $k_{0}=1$ и $A_{l} t$ имел бы степень вырожденности 0 . Но тогда вследствие симплексной итерации мы получили бы $a_{00}{ }^{t+1}<a_{00}{ }^{t}$, что противоречит предположению $t>t_{0}$. Значит, если $t>t_{0}$, то $a_{10} t \geqslant 0$. Так как $a_{10} t$ может только уменьшаться и при этом на целое число, то, начиная с некоторого $t_{1} \geqslant t_{0}$, величина $a_{10}{ }^{t}$ также остается постоянной, т. е. $a_{00}{ }^{t+1}=a_{00}{ }^{t}$ и $a_{10}{ }^{t+1}=a_{10} t \geqslant 0$ при $t \geqslant t_{1}$. Аналогичные рассуждения можно провести с компонентами $a_{20}{ }^{t}, a_{30}{ }^{t}, \ldots, a_{n 0}{ }^{t}$. Получим, что после конечного числа итераций они все станут неотрицательными целыми числами. Теорема доказана.

Правило выбора производящего ограничения, содержащееся в теореме, будем называть обобщенным правилом. Строка, выбранная по основному правилу Гомори, всегда может быть выбрана и по обобщенному правилу.

Условия теоремы можно ослабить, требуя выбора производящей строки по обобщенному правилу не на каждой итерации, а по крайней мере через каждые $q$ итераций, где $q$ - произвольное фиксированное число. Но вряд ли такой выбор имеет практическое значение.

Легко проверить, что применение обобщенного правила обеспечит конечность также вышеупомянутой модификации Ю. Ю. Финкельштейна.

В . Б. Ферстер $\left[{ }^{4}\right]$ использует для ускорения полностью целочисленного алгоритма Гомори т. н. элементарные усиленные отсечения. Но получаемые отсечения могут определить ведущий столбец высокого порядка вырожденности и, как показывает пример в. $\left[{ }^{4}\right]$, это может даже испортить конечность алгоритма. Чтобы этого не произошло, в $\left[{ }^{4}\right]$ вводится дополнительное правило выбора производящей строки. Неконечность алгоритма без этого правила объясняется тем, что элементарные усиленные отсечения могут не удовлетворять нашему обобщенному правилу (в примере $\left[{ }^{4}\right]$ это именно так). Поэтому можно рекомендовать также такой вариант модификации В. Б. Ферстера [ $\left.{ }^{4}\right]$, в котором элементарные усиленные отсечения используют только тогда, когда они удовлетворяют обобщенному правилу выбора производящей строки. 
1. Іомори Р. Е. В кн.: Календарное планирование. М., «Прогресс», 1966, 227-240.

2. Корбут А. А., Финкельштейн Ю. Ю. Дискретное программирование. М., «Наука», 1969.

3. $Х$ у T. Целочисленное программирование и потоки в сетях. М., «Мир», 1974.

4. Ферстер В. Б. В кн.: Исследование по дискретной оптимизации. М., «Наука», 1976, $53-67$.

Тартуский государственный университет

Поступила в редакцию 16/V 1983

\section{KIVISTIK}

\section{GENEREERIVA REA OLDISTATUD VALIKUREEGEL GOMORY TÄIELIKULT TÄISARVULISE ALGORITMI JAOKS}

Artiklis on esitatud Gomory täielikult täisarvulise algoritmi jaoks uus, senistest märksa paindlikum reegel genereeriva rea valikuks ja tõestatud, et algoritm on selle reegli kasutamisel lõplik.

\section{KIVISTIK}

\section{A GENERALIZED RULE OF SELECTION OF A GENERATING ROW FOR GOMORY'S ALL-INTEGER ALGORITHM}

In this paper the following integer programming problem is considered -

subject to

$$
\max x_{0}=a_{00}+\sum_{j \in J} a_{0 j}\left(-x_{j}\right) \text {, }
$$

$$
\begin{gathered}
x_{i}=a_{i 0}+\sum_{j \in J} a_{i j}\left(-x_{j}\right) \geqslant 0 \quad(i \in I), \\
x_{j}=0+(-1)\left(-x_{j}\right) \geqslant 0 \quad(j \in J), \\
x_{j} \text { is integer }(j \in I \cup J \cup\{0\} \equiv\{0,1, \ldots, n\}) .
\end{gathered}
$$

The non-negative integer $d$ is called the degeneracy degree of the vector $A_{j}=$ $=\left(a_{0 j}, a_{1 j}, \ldots, a_{n j}\right)^{\mathrm{T}}$ if its coordinates satisfy the conditions $a_{0 j}=\ldots=a_{d-1, j}=0$ and $a_{d j} \neq 0$. There is proved the following

Theorem. Assume that the simplex tableau of the problem (1)-(4) is lexicographically dually feasible and this problem has at least one plan. Then, if we choose for the generating row in every iteration step such a (given or surrogate) constraint that the degeneracy degree of the pivot column is smaller than

$$
k_{0}=\min \left\{i \in I \mid a_{i 0}<0\right\} \text {, }
$$

the Gomory's all-integer algorithm is finite. 